\title{
Precision Measurements in the Higgs Sector at ATLAS and CMS
}

\author{
André Sopczak on behalf of the ATLAS and CMS Collaborations* \\ IEAP CTU in Prague \\ E-mail: andre.sopczakecern.ch
}

\begin{abstract}
A concise review of precision measurements in the Higgs sector of the Standard Model (SM) of particle physics is given using ATLAS and CMS data. The results are based on LHC Run-2 data, taken between 2015 and 2018. Impressive progress has been made since the discovery of the Higgs boson in 2012 for measuring all major production and decay modes. Good agreement with the SM predictions was observed in all measurements.
\end{abstract}

International Conference on Precision Physics and Fundamental Physical Constants - FFK2019

9-14 June, 2019

Tihany, Hungary

${ }^{*}$ Speaker. 


\section{Introduction}

The Higgs boson of the SM is produced at the Large Hadron Collider (LHC) at CERN in different production modes. They are given below together with the production cross-sections [1] and the numbers of expected Higgs boson events for $140 \mathrm{fb}^{-1}$ corresponding to the complete Run-2 data set:

- gluon-gluon fusion (ggF), $48.58 \mathrm{pb}, 6.80 \mathrm{M}$ events,

- vector boson fusion (VBF), $3.783 \mathrm{pb}, 530 \mathrm{k}$ events,

- vector boson associated production $(\mathrm{WH}+\mathrm{ZH}),(1.415+0.8915) \mathrm{pb}, 323 \mathrm{k}$ events, and

- top-top Higgs (ttH) production, $0.507 \mathrm{pb}, 71 \mathrm{k}$ events.

The decay branching ratios of the Higgs boson in the SM are given in Table 1.

Table 1: SM Higgs boson decay branching ratios.

\begin{tabular}{c|cccccc}
\hline \hline$H \rightarrow$ & $b b$ & $W W^{*}$ & $\tau \tau$ & $Z Z^{*}$ & $\gamma \gamma$ & $\mu \mu$ \\
\hline $\mathrm{B}(\%)$ & 58 & 21 & 6.3 & 2.6 & 0.23 & 0.022 \\
\hline \hline
\end{tabular}

ATLAS [2] and CMS [3] measured the Higgs boson properties as outlined in the following sections.

\section{Higgs boson mass and width}

The Higgs boson mass has been determined by ATLAS $m_{H}=124.97 \pm 0.24 \mathrm{GeV}[4]$ and CMS $m_{H}=125.35 \pm 0.15 \mathrm{GeV}$ [5]. Indirect limits on the Higgs boson width are set at 95\% CL by ATLAS $\Gamma_{H}<14.4 \mathrm{MeV}[6]$ and $\mathrm{CMS} \Gamma_{H}<9.16 \mathrm{MeV}$ [7].

\section{Higgs boson couplings to bosons}

3.1 $H \rightarrow \gamma \gamma$

ATLAS and CMS measured the coupling $H \rightarrow \gamma \gamma$. ATLAS obtains for $79.8 \mathrm{fb}^{-1}$ data, $\mathrm{ggF}$, VBF, VH and ttH production a $\sigma \times B$ value consistent with the SM expectation [8]. Using $36.9 \mathrm{fb}^{-1}$ data, CMS measured the total Higgs boson production cross-section $\sigma_{\text {tot }}=61.1 \pm 6.0$ (stat) \pm 3.7 (syst) pb based on a combination of the $H \rightarrow \gamma \gamma$ and $H \rightarrow Z Z$ channels, which is consistent with the $\mathrm{SM}$ value $\sigma_{\mathrm{tot}}^{\mathrm{SM}}=55.6 \pm 2.5 \mathrm{pb}[9]$.

\section{2 $H \rightarrow Z Z$}

A clear signal over background is observed for $H \rightarrow Z Z \rightarrow 4 l$ for $79.8 \mathrm{fb}^{-1}$ ATLAS data [8] and $137.1 \mathrm{fb}^{-1} \mathrm{CMS}$ data [10]. For the ggF production, the $\sigma \times B$ measurement has an uncertainty of about $15 \%$. The measurement is consistent with the SM expectation. 


\section{3 $H \rightarrow W W$}

In the ATLAS $H \rightarrow W W$ analyses, the following results are obtained for ggF production $\sigma \times$ $B=11.4_{-1.1}^{+1.2}$ (stat) ${ }_{-1.1}^{+1.2}$ (theo, syst $)_{-1.3}^{+1.4}$ (exp, syst) pb, which is consistent with the SM expectation $10.4 \pm 0.6 \mathrm{pb}$. For VBF production $\sigma \times B=0.5_{-0.22}^{+0.24}$ (stat) \pm 0.10 (theo, syst) ${ }_{-0.13}^{+0.12}$ (exp, syst) pb, which is also consistent with the SM expectation of $0.81 \pm 0.02 \mathrm{pb}$ [11].

\section{Higgs boson couplings to fermions}

\section{1 $H \rightarrow \tau \tau$}

In combined ATLAS and CMS data (LHC Run-1), the observation (expectation) ${ }^{1}$ was 5.5 (5.0) st. dev. [12]. ATLAS Run-1 and $36 \mathrm{fb}^{-1}$ Run-2 data led to 6.4 (5.4) st. dev. [13]. In ggF production, the measurement is $3.1 \pm 0.1$ (stat) ${ }_{-0.13}^{+0.16}$ (syst) pb, compared to the SM expectation of $3.05 \pm 0.13 \mathrm{pb}$. For VBF production, the measurement is $0.28 \pm 0.09$ (stat) ${ }_{-0.09}^{+0.11}$ (syst) pb, compared to the SM value $0.237 \pm 0.006 \mathrm{pb}$. Similar results are obtained by CMS for $35.9 \mathrm{fb}^{-1}$ data [14], leading to 4.9 (4.7) st. dev. and 5.9 (5.9) st. dev. when combined with Run-1 data.

Stage-1 simplified template cross-sections (STXS) [15] are based on about $77.4 \mathrm{fb}^{-1} \mathrm{CMS}$ data, taken 2015-2017: $\sigma(p p \rightarrow H) \times B(H \rightarrow \tau \tau)=2.56 \pm 0.48$ (stat) \pm 0.34 (syst) pb, $\sigma(g g \rightarrow H, b b H) \times B(H \rightarrow \tau \tau)=1.11 \pm 0.81($ stat $) \pm 0.78$ (syst) pb, and $\sigma(\mathrm{VBF}) \times B(H \rightarrow \tau \tau)=0.34 \pm 0.08$ (stat) \pm 0.09 (syst) pb.

The CP invariance of the Higgs boson coupling to vector bosons has been tested in the VBF $H \rightarrow \tau \tau$ process in $36.1 \mathrm{fb}^{-1}$ ATLAS data. No evidence of $\mathrm{CP}$ violation is observed, consistent with the SM expectation [16].

\section{2 $H \rightarrow b b$}

This is a difficult channel due to large backgrounds, despite the large branching ratio (58\%). The most sensitive production mode is VH, and combined ATLAS Run- 1 and $79.8 \mathrm{fb}^{-1}$ Run-2 data yield a sensitivity of 5.4 (5.5) st. dev. [17]. Stage-1 simplified template cross-sections times $H \rightarrow b b$ branching were reported [18]. Dedicated CMS searches in ttH, VBF, ggH, and VH production modes led to 5.6 (5.5) st. dev. and $\mu=1.04 \pm 0.20$, where the signal strength $\mu$ is defined as the ratio of the measured cross-section to the SM prediction [19].

\section{$4.3 \mathrm{ttH}$}

$t t H$ observation in ATLAS has 5.8 (4.9) st. dev. [20] and in CMS has 5.2 (4.2) st. dev. [19]. The full Run-2 $139 \mathrm{fb}^{-1}$ data-set was analysed for $t t H(H \rightarrow \gamma \gamma)$ [21]. Separate event selections are applied corresponding to the decay modes of the top quarks (hadronic and leptonic). Templates from top mass distributions were constructed in $t t \gamma \gamma, \gamma \gamma+\mathrm{jets}$, and $t t H$ simulations in order to decompose the continuum background by a template fit to the data.

The CMS observation with 2016 data was 5.2 (4.2) st. dev. (combined $b b$, multilepton, $\gamma \gamma, Z Z$ channels) [22], including 2017 data, the analysis of $t+H(H \rightarrow \gamma \gamma)$ resulted in $\mu=1.7_{-0.5}^{+0.6}$ [23].

In the $t t H$ multilepton channels with $\ell=e$ or $\mu$, and $\tau$ (hadronic decay) $\mu=0.96_{-0.31}^{+0.34}\left(1.00_{-0.27}^{+0.30}\right)$ for $35.9 \mathrm{fb}^{-1}$ (2016 CMS data) was obtained [23]. For $41.5 \mathrm{fb}^{-1}$ (2017 ATLAS data) 3.2 (4.0) st. dev. was achieved [24]. In an analysis of $t t H$ and $t t W$ production in multilepton final states using $80 \mathrm{fb}^{-1}$, ATLAS measured the $t t H$ signal with 1.8 (3.1) st. dev. above the SM background [25].

\footnotetext{
${ }^{1}$ The expected significance is given in parenthesis throughout the article.
} 
The $t t H(H \rightarrow b b)$ fully hadronic, single-lepton and double-lepton final states were analysed by CMS, leading to 3.7 (2.6) st. dev. [26].

4.4 $H \rightarrow \mu \mu$

As the $\mathrm{SM} B(H \rightarrow \mu \mu)=0.022 \%$ is very small, currently, only limits are set at $95 \% \mathrm{CL}$ on $\mu=\sigma(p p \rightarrow H) \times B(H \rightarrow \mu \mu) / \sigma(p p \rightarrow H)_{\mathrm{SM}} \times B(H \rightarrow \mu \mu)_{\mathrm{SM}}<2.1$ (2.0) by ATLAS [27] and $<2.9$ (2.2) by CMS [28].

\section{Simplified template cross-sections STXS}

STXS was proposed at the Les Houches' 15 workshop and by the LHC Higgs boson crosssection working group. The goal was to have a common format for ATLAS, CMS and theory, in particular, to measure cross-sections per production modes $(\mathrm{ggF}, \mathrm{VBF}, \mathrm{VH}, \mathrm{ttH})$ in different phase space, signal templates $p_{\mathrm{T}}(H), p_{\mathrm{T}}(V)$, etc., reducing model dependency and maximizing sensitivity to BSM effects, and to combine different decay channels in order to increase sensitivity.

An ATLAS combination of the main channels was performed with STXS stage-1 [29]. In STXS, several channels contribute to different kinematic regions of the same production mode, e.g., $\mathrm{VH}$ dominated by $H \rightarrow b b$ in high $p_{\mathrm{T}}(V)$, while $g g$ and $Z Z^{*}$ are relevant at low $p_{\mathrm{T}}(V)$. No significant deviation from SM predictions in any kinematic region was observed, and the p-value with respect to the SM hypothesis is 0.80 .

A CMS combination of various production modes and decay channels was performed with $35.9 \mathrm{fb}^{-1}$ [30]. A first CMS measurement of STXS stage-1 regions in the diphoton channel was performed, covering the gluon fusion $(\mathrm{ggH})$ and vector boson fusion (VBF) production modes. For STXS stage-1, ggH and VBF bins using $H \rightarrow \gamma \gamma$ based on $77.4 \mathrm{fb}^{-1}$ were analysed [31]. Ten ggH and three VBF parameters were defined, depending on the number of jets and $p_{\mathrm{T}}(H)$. For each of the thirteen signal parameters, the measured cross-section was compared with the SM prediction, and good agreement was obtained.

\section{Differential Higgs boson decay cross-sections}

Differential Higgs boson decay cross-sections have been measured for several modes, e.g., $H \rightarrow \gamma \gamma$ with ATLAS data [32] and $H \rightarrow \gamma \gamma, Z Z, 4 \ell, b b$ combined modes with CMS data [9].

\section{Rare Higgs boson decays}

Further SM Higgs boson decay modes can be in reach with growing LHC data sets. Current ATLAS limits at $95 \%$ CL are:

- $\mu=\sigma(p p \rightarrow H) \times B(H \rightarrow Z \gamma) / \sigma(p p \rightarrow H) \times B(H \rightarrow Z \gamma)_{S M}<6.6$ (4.4) [33], and

- $\mu=\sigma_{\mathrm{HZ}} \times B(H \rightarrow c c) / \sigma_{\mathrm{HZ}} \times B(H \rightarrow c c)_{S M}<110(150)$ [34].

The four-muon final state is experimentally clean containing only very small SM background [35], however, the observed (expected) CMS limits are far from the expected SM rates. The limits at 95\% CL are: 
- $B(H \rightarrow J / \psi J / \psi)<1.8 \cdot 10^{-3}$ (obs), $<\left(1.8_{-0.1}^{+0.2}\right) \cdot 10^{-3}$ (exp), and

- $B(H \rightarrow \Upsilon \Upsilon)<1.4 \cdot 10^{-3}$ (obs),$<(1.4 \pm 0.1) \cdot 10^{-3}$ (exp).

Anomalous couplings were also searched for [7].

\section{Higgs boson decays into invisible particles}

A motivation for this search was given by Patt and Wilczek, "Higgs-field portal into hidden sectors" [36]. There are indirect constraints from coupling fits, and direct constraints from searches for Higgs bosons decaying into invisible particles. Three separate ATLAS searches were performed: $V$ (had) $H$ (inv), $Z$ (lep) $H$ (inv) and VBF $H($ inv), with the result $B(H \rightarrow$ inv $)<0.26(0.17)$ at $95 \% \mathrm{CL}$, assuming SM production cross-section [37].

For CMS, the dominant backgrounds are $Z(v v)+$ jets and $W(\ell v)+$ jets, extrapolated from 2-lepton sideband, and from 1-lepton sideband, respectively. The VBF production channel is the most sensitive one. 2016 VBF-only data led to $B(H \rightarrow$ inv $)<0.33(0.25)$ at $95 \% \mathrm{CL}$.

About $25 \%$ improvement in sensitivity is obtained by adding $\mathrm{VH}$ and $\mathrm{ggH}$ channels, thus $B(H \rightarrow$ inv $)<0.26(0.20)$ at $95 \% \mathrm{CL}(13 \mathrm{TeV}$ data $)$, and $B(H \rightarrow$ inv $)<0.19(0.15)$ using 7,8 , $13 \mathrm{TeV}$ data [38]. $t t H$ limits on invisible decays are also set at 95\% CL $B(H \rightarrow$ inv $)<0.46(0.48)$ [39].

\section{Combination}

The ATLAS results of the measurements from several production and decay modes were combined taking their uncertainties into account [29]. The result is expressed as a comparison to the SM expectation $\mu=1.11_{-0.08}^{+0.09}$. Thus, the combined result is compatible with the expectation from the SM.

\section{Higgs boson production modes (ggF, VBF, VH, ttH)}

All major Higgs boson production modes are observed ( $>5$ st. dev.) based on $79.8 \mathrm{fb}^{-1}$ ATLAS data, assuming SM branching ratios [29]:

- $\mathrm{ggF}, \mathrm{VBF}$ (6.5 st. dev.),

- VH (5.3 st. dev.), and

- $\mathrm{ttH}(5.8$ st. dev.).

There are only small correlations between production modes, and the results are consistent with the SM expectations. Similar results are obtained by a CMS data combination [30].

Cross-sections are measured for VBF versus ggF. Individual and combined decay modes are in agreement with the SM expectation [29]. A generic parametrization of the measured couplings with respect to the SM was performed for $\lambda(t g)$ contributing through ggF loop, as compared to $\mathrm{ttH}$, and $\lambda(\gamma Z)$ contributing to the $H \rightarrow \gamma \gamma$ loop, as compared to $H \rightarrow Z Z$ decays. The results are also in agreement with SM expectations.

For $137 \mathrm{fb}^{-1}$ data in the $H \rightarrow Z Z \rightarrow 4 \ell$ channel $(\ell=e$ or $\mu$ ), the cross-section measurement is $\sigma=2.73_{-0.22}^{+0.23}$ (stat) ${ }_{-0.19}^{+0.24}$ (syst) fb, which is consistent with the SM expectation $2.76 \pm 0.14 \mathrm{fb}$ [10]. Differential cross-sections versus $p_{\mathrm{T}}(H), H$ rapidity, and the number of jets were also studied. 


\section{Single-top-Higgs production tH, Higgs boson pair-production HH}

The single-top-Higgs production $t H(H \rightarrow W W, Z Z, \tau \tau, b b)$ was searched for. A combination with $t t H$ and $H \rightarrow \gamma \gamma$ could give sensitivity to the absolute values of the top quark Yukawa coupling and the Higgs boson coupling to vector bosons $g_{H V V}$, and uniquely, to their relative sign. The SM-like signal favours a $\kappa_{t}=1.0$ over $\kappa_{t}=-1.0$ by $>1.5$ st. dev. [40]. ATLAS sets a limit on $H H \rightarrow b b b b, b b \tau \tau, b b \gamma \gamma<6.7(10.4) \times \mathrm{SM}$ at $95 \%$ CL. CMS obtained a limit $H H \rightarrow b b V V, b b b b, b b \tau \tau, b b \gamma \gamma<22.2(12.8) \times \mathrm{SM}$ at 95\% CL [41].

\section{Relation of coupling and fermion mass}

An interpretation of the results is given in the $\kappa$-framework as a function of the particle mass, assuming only SM contributions to the total width [29, 30]. Figure 1 shows the linearity as expected from the Higgs boson theory for ATLAS and CMS. Under the assumption that new physics affects only the Higgs boson self-coupling, the best fit value of the coupling modifier is $\kappa_{\lambda}=4.6_{-3.8}^{+3.2}$, excluding values outside the interval $-2.3<\kappa_{\lambda}<10.3$ at $95 \% \mathrm{CL}$, while the expected excluded range assuming the $\mathrm{SM}$ predictions is $-5.1<\kappa_{\lambda}<11.2$ [42].
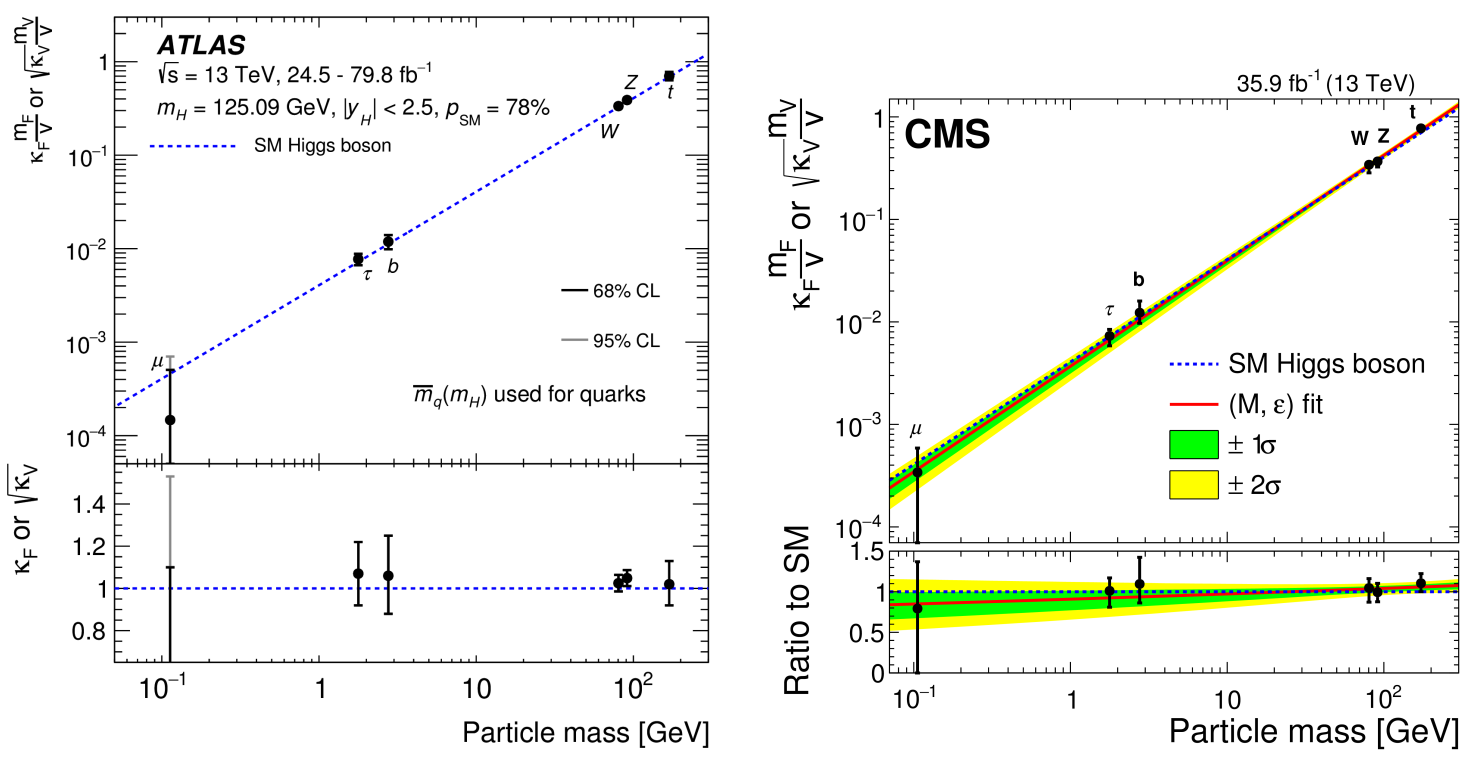

Figure 1: Left (ATLAS result) [29]: Reduced coupling-strength modifiers $\kappa_{F} m_{F} / v$ for fermions $(F=$ $t, b, \tau, \mu)$ and $\sqrt{\kappa_{V}} m_{V} / v$ for weak gauge bosons $(V=W, Z)$ as a function of their masses $m_{F}$ and $m_{V}$, respectively, and the vacuum expectation value of the Higgs field $v=246 \mathrm{GeV}$. The SM prediction for both cases is also shown (dotted line). The black error bars represent $68 \% \mathrm{CL}$ intervals for the measured parameters. For $\kappa_{\mu}$ the light error bars indicate the $95 \%$ CL interval. The coupling modifiers $\kappa_{F}$ and $\kappa_{V}$ are measured assuming no BSM contributions to the Higgs boson decays, and assuming the SM structure of loop processes such as $g g \rightarrow H, H \rightarrow \gamma \gamma$ and $H \rightarrow g g$. The lower panel shows the ratios of the values to their SM predictions. Right (CMS result) [30]: Result of the phenomenological $(M, \varepsilon)$ fit is also overlayed with the resolved $\kappa$-framework model. 


\section{Conclusions and Outlook}

In conclusion, LHC Run-2 operation was very successful. Observations of the Higgs to tau coupling, Higgs to bottom coupling, and Higgs to top coupling were reported. Sensitivity to the Higgs to muon coupling is approaching. After establishing these inclusive measurements, the focus is now on differential measurements and combinations of all main LHC Higgs boson production modes. So far, all Higgs boson properties are in agreement with the SM expectations.

The outlook for LHC Run-2 data analysis is towards a complete analysis of the LHC Run-2 data set (about $140 \mathrm{fb}^{-1}$ per experiment), a more detailed understanding of the data to increase the measurement precision, and a combination of ATLAS and CMS results to increase sensitivities further. LHC Run-3 is anticipated to add $300 \mathrm{fb}^{-1}$ (during the 2021 to 2023 data-taking), and HL-LHC is approved for $3000 \mathrm{fb}^{-1}$ (expected to start delivering data in 2026) for a new era of measurements with higher precision. Overall there is a strong and approved LHC programme for new discoveries and further precision measurements.

\section{Acknowledgments}

I would like the thank the colleagues from the ATLAS and CMS Higgs working groups, and the theorists and phenomenologists present at the conference for the fruitful discussions, as well as the organizers of FFK2019 for their invitation and hospitality. The project is supported by the Ministry of Education, Youth and Sports of the Czech Republic under project number LTT17018.

\section{References}

[1] LHC Higgs Cross Section Working Group collaboration, Handbook of LHC Higgs Cross Sections: 4. Deciphering the Nature of the Higgs Sector, CERN Yellow Reports: Monographs. Oct, 2016, 10.23731/CYRM-2017-002.

[2] ATLAS collaboration, The ATLAS Experiment at the CERN Large Hadron Collider, JINST 3 (2008) S08003. 437 p.

[3] CMS collaboration, The CMS experiment at the CERN LHC. The Compact Muon Solenoid experiment, JINST 3 (2008) S08004. 361 p.

[4] ATLAS collaboration, Measurement of the Higgs boson mass in the $H \rightarrow Z Z^{*} \rightarrow 4 \ell$ and $H \rightarrow \gamma \gamma$ channels with $\sqrt{s}=13 \mathrm{TeV}$ pp collisions using the ATLAS detector, Phys. Lett. B 784 (2018) 345.

[5] CMS collaboration, A measurement of the Higgs boson mass in the diphoton decay channel, CMS-PAS-HIG-19-004, CERN, Geneva, 2019.

[6] ATLAS collaboration, Constraints on off-shell Higgs boson production and the Higgs boson total width in $Z Z \rightarrow 4 \ell$ and $Z Z \rightarrow 2 \ell 2 v$ final states with the ATLAS detector, Phys. Lett. B 786 (2018) 223.

[7] CMS collaboration, Measurements of the Higgs boson width and anomalous HVV couplings from on-shell and off-shell production in the four-lepton final state, Phys. Rev. D 99 (2019) 112003. 33 p.

[8] ATLAS collaboration, Measurements of the Higgs boson production, fiducial and differential cross sections in the $4 \ell$ decay channel at $\sqrt{s}=13 \mathrm{TeV}$ with the ATLAS detector, ATLAS-CONF-2018-018, CERN, Geneva, Jun, 2018. 
[9] CMS collaboration, Measurement and interpretation of differential cross sections for Higgs boson production at $\sqrt{s}=13 \mathrm{TeV}$, Phys. Lett. B 792 (2018) 369. $28 \mathrm{p}$.

[10] CMS collaboration, Measurements of properties of the Higgs boson in the four-lepton final state in proton-proton collisions at $\sqrt{s}=13 \mathrm{TeV}$, CMS-PAS-HIG-19-001, CERN, Geneva, 2019.

[11] ATLAS collaboration, Measurements of gluon-gluon fusion and vector-boson fusion Higgs boson production cross-sections in the $H \rightarrow W W^{*} \rightarrow e v \mu v$ decay channel in pp collisions at $\sqrt{s}=13 \mathrm{TeV}$ with the ATLAS detector., Phys. Lett. B 789 (2018) 508.

[12] ATLAS AND CMS collaboration, Measurements of the Higgs boson production and decay rates and constraints on its couplings from a combined ATLAS and CMS analysis of the LHC pp collision data at $\sqrt{s}=7$ and $8 \mathrm{TeV}$, JHEP 08 (2016) $045.70 \mathrm{p}$.

[13] ATLAS collaboration, Cross-section measurements of the Higgs boson decaying into a pair of $\tau$-leptons in proton-proton collisions at $\sqrt{s}=13 \mathrm{TeV}$ with the ATLAS detector, Phys. Rev. D 99 (2018) $072001.58 \mathrm{p}$.

[14] CMS collaboration, Observation of the Higgs boson decay to a pair of $\tau$ leptons with the CMS detector, Phys. Lett. B 779 (2017) 283.

[15] CMS collaboration, Measurement of Higgs boson production and decay to the $\tau \tau$ final state, CMS-PAS-HIG-18-032, CERN, Geneva, 2019.

[16] ATLAS collaboration, Test of CP invariance in vector-boson fusion production of the Higgs boson in the $H \rightarrow \tau \tau$ channel in proton-proton collisions at $\sqrt{s}=13$ TeV with the ATLAS detector, ATLAS-CONF-2019-050, CERN, Geneva, Oct, 2019.

[17] ATLAS collaboration, Observation of $H \rightarrow b \bar{b}$ decays and VH production with the ATLAS detector, Phys. Lett. B 786 (2018) 59.

[18] ATLAS collaboration, Measurement of $V H, H \rightarrow b \bar{b}$ production as a function of the vector-boson transverse momentum in $13 \mathrm{TeV}$ pp collisions with the ATLAS detector, JHEP 1905 (2019) 141. 35 p.

[19] CMS collaboration, Observation of Higgs boson decay to bottom quarks, Phys. Rev. Lett. 121 (2018) 121801. $20 \mathrm{p}$.

[20] ATLAS collaboration, Observation of Higgs boson production in association with a top quark pair at the LHC with the ATLAS detector, Phys. Lett. B 784 (2018) 173.

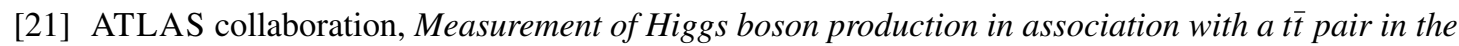
diphoton decay channel using $139 \mathrm{fb}^{-1}$ of LHC data collected at $\sqrt{\mathrm{s}}=13 \mathrm{TeV}$ by the ATLAS experiment, ATLAS-CONF-2019-004, CERN, Geneva, Mar, 2019.

[22] CMS collaboration, Observation of $\mathrm{t} \overline{\mathrm{t}} H$ production, Phys. Rev. Lett. 120 (2018) 231801. 17 p.

[23] CMS collaboration, Measurement of the associated production of a Higgs boson and a pair of top-antitop quarks with the Higgs boson decaying to two photons in proton-proton collisions at $\sqrt{s}=13$ TeV, CMS-PAS-HIG-18-018, CERN, Geneva, 2018.

[24] ATLAS collaboration, Evidence for the associated production of the Higgs boson and a top quark pair with the ATLAS detector, Phys. Rev. D 97 (2017) 072003. 44 p.

[25] ATLAS collaboration, Analysis of $t \bar{t} H$ and $t \bar{t} W$ production in multilepton final states with the ATLAS detector, ATLAS-CONF-2019-045, CERN, Geneva, Oct, 2019.

[26] CMS collaboration, Measurement of $\mathrm{t} \overline{\mathrm{t}} \mathrm{H}$ production in the $\mathrm{H} \rightarrow \mathrm{b} \overline{\mathrm{b}}$ decay channel in $41.5 \mathrm{fb}^{-1}$ of proton-proton collision data at $\sqrt{s}=13 \mathrm{TeV}$, CMS-PAS-HIG-18-030, CERN, Geneva, 2019. 
[27] ATLAS collaboration, A search for the rare decay of the Standard Model Higgs boson to dimuons in pp collisions at $\sqrt{s}=13 \mathrm{TeV}$ with the ATLAS Detector, ATLAS-CONF-2018-026, CERN, Geneva, Jul, 2018.

[28] CMS collaboration, Search for the Higgs boson decaying to two muons in proton-proton collisions at $\sqrt{s}=13 \mathrm{TeV}$. Search for the Higgs boson decaying to two muons in proton-proton collisions at $\sqrt{s}=$ 13 TeV, Phys. Rev. Lett. 122 (2018) 021801. 18 p.

[29] ATLAS collaboration, Combined measurements of Higgs boson production and decay using up to 80 $\mathrm{fb}^{-1}$ of proton-proton collision data at $\sqrt{\mathrm{s}}=13 \mathrm{TeV}$ collected with the ATLAS experiment, arXiv:1909.02845, CERN, Geneva, Sep, 2019.

[30] CMS collaboration, Combined measurements of Higgs boson couplings in proton-proton collisions at $\sqrt{s}=13$ TeV, Eur. Phys. J. C 79 (2018) 421.67 p.

[31] CMS collaboration, Measurements of Higgs boson production via gluon fusion and vector boson fusion in the diphoton decay channel at $\sqrt{s}=13 \mathrm{TeV}$, CMS-PAS-HIG-18-029, CERN, Geneva, 2019.

[32] ATLAS collaboration, Measurements of Higgs boson properties in the diphoton decay channel using $80 \mathrm{fb}^{-1}$ of pp collision data at $\sqrt{\mathrm{s}}=13 \mathrm{TeV}$ with the ATLAS detector, ATLAS-CONF-2018-028, CERN, Geneva, Jul, 2018.

[33] ATLAS collaboration, Searches for the Z $\gamma$ decay mode of the Higgs boson and for new high-mass resonances in pp collisions at $\sqrt{s}=13 \mathrm{TeV}$ with the ATLAS detector, JHEP 10 (2017) 112. $52 \mathrm{p}$.

[34] ATLAS collaboration, Search for the Decay of the Higgs Boson to Charm Quarks with the ATLAS Experiment, Phys. Rev. Lett. 120 (2018) 211802. 20 p.

[35] CMS collaboration, Search for Higgs and Z boson decays to J/ $\psi$ or $\Upsilon$ pairs in proton-proton collisions at $\sqrt{s}=13 \mathrm{TeV}$, CMS-PAS-HIG-18-025, CERN, Geneva, 2019.

[36] B. Patt and F. Wilczek, Higgs-field portal into hidden sectors, hep-ph/0 605188.

[37] ATLAS collaboration, Combination of searches for invisible Higgs boson decays with the ATLAS experiment, ATLAS-CONF-2018-054, CERN, Geneva, Nov, 2018.

[38] CMS collaboration, Search for invisible decays of a Higgs boson produced through vector boson fusion in proton-proton collisions at $\sqrt{s}=13 \mathrm{TeV}$, Phys. Lett. (2018) $32 \mathrm{p}$.

[39] CMS collaboration, First constraints on invisible Higgs boson decays using t $\mathrm{t} H$ production at $\sqrt{s}=13 \mathrm{TeV}$, CMS-PAS-HIG-18-008, CERN, Geneva, 2019.

[40] ATLAS collaboration, Combination of searches for Higgs boson pairs in pp collisions at $\sqrt{s}=13$ TeV with the ATLAS detector, arXiv:1906.02025, CERN, Geneva, Jun, 2019.

[41] CMS collaboration, Combination of searches for Higgs boson pair production in proton-proton collisions at $\sqrt{s}=13 \mathrm{TeV}$, Phys. Rev. Lett. 122 (2018) 121803. 18 p.

[42] ATLAS collaboration, Constraints on the Higgs boson self-coupling from the combination of single-Higgs and double-Higgs production analyses performed with the ATLAS experiment, ATLAS-CONF-2019-049, CERN, Geneva, Oct, 2019. 